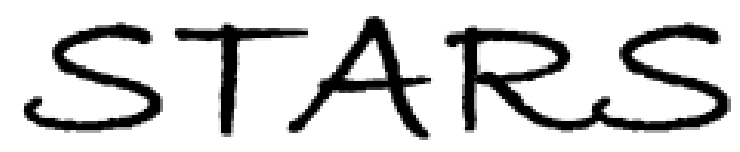

University of Central Florida

STARS

$1-1-1996$

\title{
One-Dimensional Spatial Solitary Waves Due To Cascaded Second-Order Nonlinearities In Planar Waveguides
}

\author{
Roland Schiek \\ University of Central Florida \\ Yongsoon Baek \\ University of Central Florida \\ George I. Stegeman \\ University of Central Florida
}

Find similar works at: https://stars.library.ucf.edu/facultybib1990

University of Central Florida Libraries http://library.ucf.edu

This Article is brought to you for free and open access by the Faculty Bibliography at STARS. It has been accepted for inclusion in Faculty Bibliography 1990s by an authorized administrator of STARS. For more information, please contactSTARS@ucf.edu.

\section{Recommended Citation}

Schiek, Roland; Baek, Yongsoon; and Stegeman, George I., "One-Dimensional Spatial Solitary Waves Due To Cascaded Second-Order Nonlinearities In Planar Waveguides" (1996). Faculty Bibliography 1990 s. 1748.

https://stars.library.ucf.edu/facultybib1990/1748

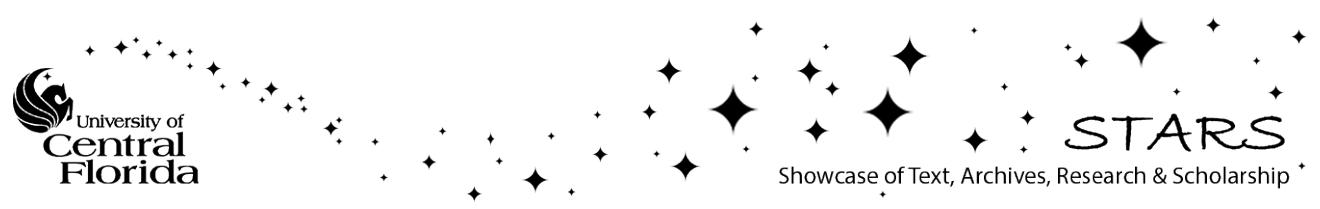




\title{
One-dimensional spatial solitary waves due to cascaded second-order nonlinearities in planar waveguides
}

\author{
Roland Schiek, Yongsoon Baek, and George I. Stegeman \\ CREOL, University of Central Florida, 12424 Research Parkway, Orlando, Florida 32826
}

(Received 1 September 1995)

\begin{abstract}
We report an experimental observation of one-dimensional spatial solitary waves due to cascaded secondorder optical nonlinearities.
\end{abstract}

PACS number(s): 42.65.Tg, 42.65.Jx, 42.65.Ky

Solitons are stable nondiffracting solutions to the nonlinear wave equation and their unique properties have intrigued physicists for many years [1]. Electromagnetic waves have provided the most versatile approach to the experimental investigation of soliton properties. One-dimensional temporal (no pulse spreading in time) and spatial (no beam spreading in space) solitons, both bright and dark, and their interactions have been investigated optically [2-4]. These phenomena have all relied upon the existence of an intensity-dependent refractive index, i.e., a third-order optical nonlinearity. That is, a high-intensity beam changes locally the index distribution, which then self-traps the beam (soliton) under appropriate conditions. Recently, another nonlinear physical process that produces a nonlinear distortion in the phase of a beam without changing its index has been revisited [5-7]. It involves one of the best-known nonlinear optical processes, namely, second-harmonic generation (SHG). The phase distortion utilizes the cascading of two nonlinear second-order processes: the up-conversion of a fundamental beam to a second harmonic and the subsequent down-conversion of the harmonic back to the fundamental. Within certain limits the equations that describe the copropagation of the beams reduce to the nonlinear wave equation for the fundamental, the same equation that leads to spatial solitons based on an intensity-dependent refractive index [1]. As a result, it has been predicted that this cascading mechanism should allow the existence of spatial solitonlike (solitary) waves in simple and apparently well-understood processes such as secondharmonic generation [7-13]. In this paper we show experimentally that such spatial solitary waves do exist in one dimension.

The geometry we consider is a planar waveguide with index differences providing guided mode confinement along the $x$ axis and propagation along the $z$ axis. The self-induced trapping will occur in the plane of the waveguide, i.e., along the $y$ axis. Perpendicular to the film the guided field distributions $e_{i}(x)$ and $h_{i}(x)$ are waveguide modes, normalized to unity power flow per meter of film width along the $y$ axis, i.e., $p_{0}=1 \mathrm{~W} / \mathrm{m}$. $i=1,2$ identifies the fundamental $\left(\omega_{1}\right)$ and second harmonic $\left(\omega_{2}=2 \omega_{1}\right)$, respectively. Parallel to the film, the $y$ dependence of the fields $E_{i}(x, y)$ at every position $z$ along the waveguide can be expanded as spatial Fourier integrals

$$
E_{i}(x, y)=\frac{e_{i}(x)}{2 \pi} \int d \beta_{y} A_{i}\left(\beta_{y}\right) \exp \left(j \beta_{y} y\right)
$$

The up- and down-conversion processes are described in coupled-mode theory as

$$
\begin{aligned}
\frac{d A_{1}\left(\beta_{y}\right)}{d z}+j \beta_{1 z} A_{1}\left(\beta_{y}\right)= & -j \frac{\omega_{1} K^{(2)} 2 \chi^{(2)} \beta_{1}}{4 p_{0} \beta_{1 z} 2 \pi} \\
& \times \int d \beta_{y}^{\prime} A_{2}\left(\beta_{y}-\beta_{y}^{\prime}\right) A_{1}^{*}\left(-\beta_{y}^{\prime}\right) \\
\frac{d A_{2}\left(\beta_{y}\right)}{d z}+j \beta_{2 z} A_{2}\left(\beta_{y}\right)= & -j \frac{2 \omega_{1} K^{(2)} \chi^{(2)} \beta_{2}}{4 p_{0} \beta_{2 z} 2 \pi} \\
& \times \int d \beta_{y}^{\prime} A_{1}\left(\beta_{y}-\beta_{y}^{\prime}\right) A_{1}\left(\beta_{y}^{\prime}\right)
\end{aligned}
$$

respectively $[7,13] . \beta_{y}$ is the spatial angular frequency and the $\beta_{i z}=\left(\beta_{i}^{2}-\beta_{y}^{2}\right)^{1 / 2}$ are the $z$ components of the mode propagation constants $\beta_{i} . K^{(2)}=\int d x e_{1} e_{1} e_{2}^{*}$ is the overlap integral, which takes into account the different transverse electric-field profiles of the interacting modes. Also, the scalar $\chi^{(2)}$ represents the appropriate tensor coefficient $\chi_{y x x}^{(2)}$. In the limit of negligible SHG, the case of experimental interest here, Eqs. (2) and (3) can be recast into a form similar to that used to describe spatial soliton propagation [7]. Assuming $A_{1}=\mathscr{b}_{1} \exp \left(-j \beta_{1} z\right)$, Eq. (3) can be integrated approximately and substituted into Eq. (2) to give

$$
\frac{\partial}{\partial z}, b_{1}+j \frac{1}{2 \beta_{1}} \frac{\partial^{2}}{\partial y^{2}}, b_{1}=-j \frac{\left[\omega_{1} K^{(2)} \chi^{(2)}\right]^{2}}{4 p_{0}^{2}\left[2 \beta_{1}-\beta_{2}\right]}\left|\cdot \ell_{1}\right|^{2} \mathscr{l}_{1} .
$$

A comparison with the nonlinear wave equation indicates that the right-hand side plays the role of an effective thirdorder nonlinearity $[1,7]$. This implies approximate solitonlike solutions for the fundamental of the form

$$
\mathscr{b}_{1}(x, y)=\left[\frac{32\left(p_{0} \eta\right)^{2}\left(2 \beta_{1}-\beta_{2}\right)}{\left(\omega_{1} \chi^{(2)} K^{(2)}\right)^{2}}\right]^{1 / 2} \frac{\exp \left(-j 4 \eta^{2} z\right)}{\cosh \left(2 \eta y\left[2 \beta_{1}\right]^{1 / 2}\right)}
$$

for positive phase mismatch $2 \beta_{1}-\beta_{2}>0 . \quad \eta$ is the beam width. The conclusion of this approximate treatment is that cascading should support spatial solitonlike waves.

We modeled the evolution of high-power guided wave beams in planar $\mathrm{LiNbO}_{3}$ waveguides based on the exact

(C) 1996 The American Physical Society 


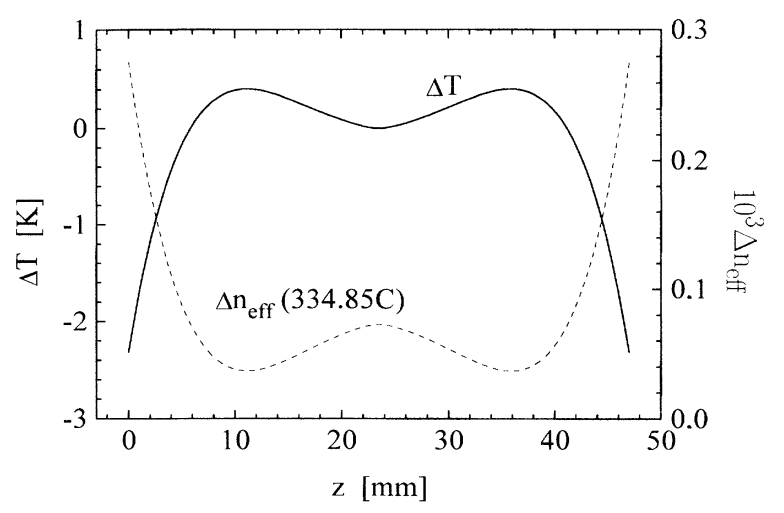

FIG. 1. Effective temperature profile $\Delta T=T(z)-T_{\text {set }}$ and wavevector-mismatch distribution $\Delta n_{\mathrm{eff}}=\left\{2 \beta_{1}[T(z)]-\beta_{2}[T(z)]\right\} /$ $\left(2 \pi / \lambda_{2}\right)$ for a setpoint temperature $T_{\text {set }}$ of $334.85^{\circ} \mathrm{C}$.

equations (2) and (3), taking into account the details of the waveguide and the experimental conditions (discussed later). In our case only the fundamental beam is input (no secondharmonic seeding) and the experimental and theoretical conditions were chosen so that SHG was minimal $(<5 \%)$ and the solitary waves studied should be well approximated by Eq. (5). We assumed cw, Gaussian input beams [70 $\mu \mathrm{m}$ full width at half maximum (FWHM)] because we found their transverse profiles to very closely match the asymptotic, stable, solitary waves for our laser power. Assuming the experimental, positive wave-vector-mismatch distribution shown in Fig. 1, the field evolution shown in Fig. 2 indicates self-trapping and self-focusing for input beams with peak transverse powers exceeding $6 \mathrm{~W} / \mu \mathrm{m}$. At $16 \mathrm{~W} / \mu \mathrm{m}$ the evolution of the field profile is essentially free of oscillations because the input Gaussian beam launches the asymptotic stable solitary wave right at the input due to the excellent transverse field overlap; see Fig. 2(b). For a fixed input beam width, the field evolution into a stable solitary wave exhibits transient oscillations along the propagation direction when the input power deviates from $16 \mathrm{~W} / \mu \mathrm{m}$. Regions of nonlinear self-focusing are followed by regions where diffraction dominates until the balance between self-focusing and diffraction leads to a stable solitary wave. When the input beam profile does not match the soliton profile such transient behavior is typical of solitons in general and can require many diffraction lengths $L_{d}$ (the distance for a diffracting beam to be broadened by $\sqrt{2}$ times its minimum width) [14]. In our case, our sample is $47 \mathrm{~mm}$ long and $L_{d}=\pi \rho_{0}^{2} n / \lambda_{1}=19 \mathrm{~mm}$ with $\rho_{0}=(\mathrm{FWHM}) /[2 \ln 2]^{1 / 2}$ and $n=2.22$. Therefore, for input beam profiles deviating strongly from the asymptotic solitary waves, or at input powers greater than $25 \mathrm{~W} / \mu \mathrm{m}$, our sample would be too short to reach the asymptotic solitarywave solutions. This was not the case in most of our experiments. The calculations were repeated for temporal pulses, assuming that the pulses are long enough so that temporal dispersion can be neglected. For our experiments this approximation is valid. The pulsed beam behavior was qualitatively the same, with an increase in peak power from 16 to $25 \mathrm{~W} / \mu \mathrm{m}$ for optimally launching spatial solitary waves.

The experiments were performed at $\lambda_{1}=1.32 \mu \mathrm{m}$ in a $\mathrm{Ti}$ indiffused $\mathrm{LiNbO}_{3}$ slab waveguide with propagation along the $x$ axis of a $Y$-cut crystal for type-I SHG.
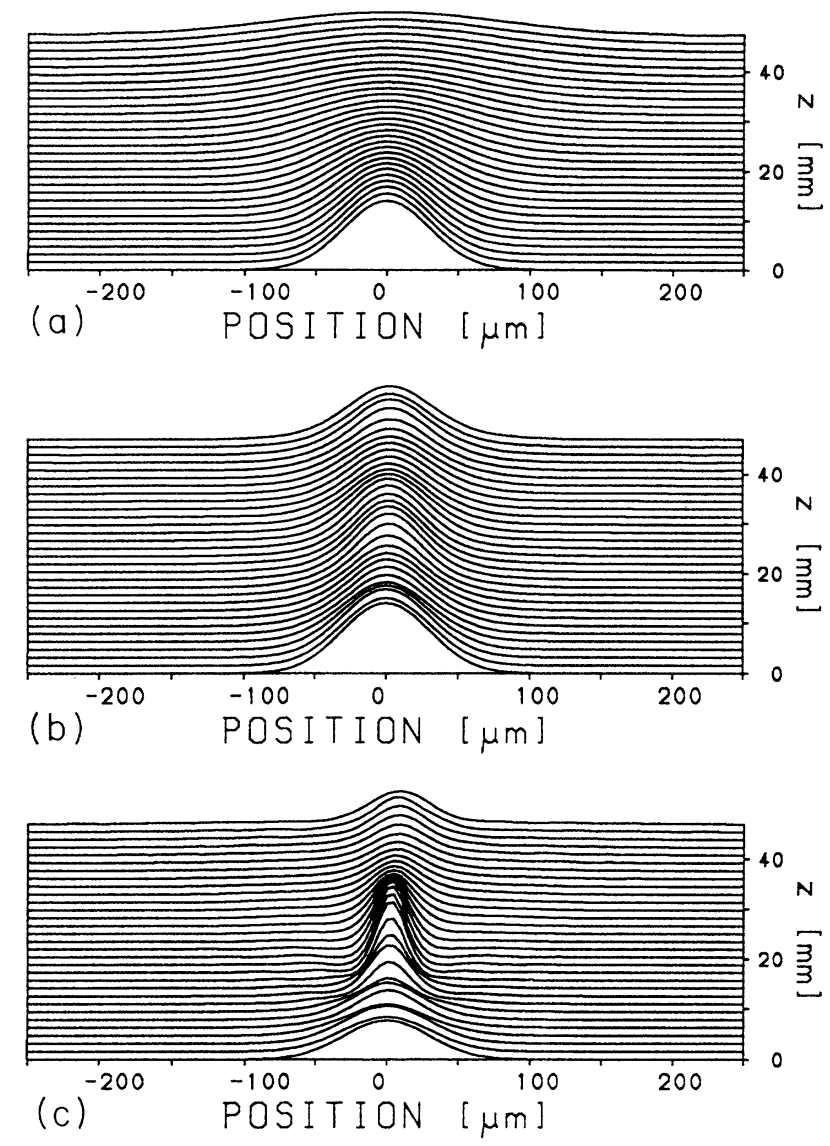

FIG. 2. Fundamental intensity along the waveguide with a temperature of $334.85{ }^{\circ} \mathrm{C}$ for different powers: (a) $7.5 \mathrm{~W}(0.1 \mathrm{~W} / \mu \mathrm{m})$, (b) $1.2 \mathrm{~kW}(16 \mathrm{~W} / \mu \mathrm{m})$, and (c) $5.3 \mathrm{~kW}(70 \mathrm{~W} / \mu \mathrm{m})$.

$\chi^{(2)}=\chi_{Z Y Y}^{(2)}(2 \omega ; \omega, \omega)=\varepsilon_{0}(-5.6 \mathrm{pm} / \mathrm{V})$ is the applicable susceptibility tensor element. The indiffusion of a $55-\mathrm{nm}$-thick titanium layer through the $Y$-cut surface at $1060^{\circ} \mathrm{C}$ for $9 \mathrm{~h}$ yielded a low-loss one-dimensional waveguide, which guides one TM mode ( $Y$ polarized) at $\lambda_{1}=1.32 \mu \mathrm{m}$ and three second-harmonic TE modes $\left(Z\right.$ polarized) at $\lambda_{2}=0.66 \mu \mathrm{m}$. With a cylindrical telescope the laser beam was transformed into an elliptical Gaussian beam, which was endfire coupled into the fundamental $\mathrm{TM}_{0}$ mode. The end faces are polished for endfire coupling with the output surface tilted at $4.5^{\circ}$ to prevent longitudinal cavity resonances. In order to generate spatial solitary waves via the cascaded nonlinearity, SHG was implemented from the $\mathrm{TM}_{0}\left(\omega_{1}\right)$ to the $\mathrm{TE}_{1}\left(2 \omega_{1}\right)$ mode, which is phase matched around $335^{\circ} \mathrm{C}$ for our material, geometry, and wavelength. For this purpose the crystal was placed in an oven with temperature controlled to a stability of $\pm 25 \mathrm{mK}$. We investigated the output beam characteristics for a $70-\mu \mathrm{m}$ FWHM input beam, both at different beam intensities and different temperatures (corresponding to different wave-vector mismatches). The out-coupling surface is imaged into a camera where the output intensity profile is measured. In addition, the fundamental throughput, the generated second-harmonic power, and the intensity in the center of the output beam were monitored with fast detectors. The measurements were done with a train of pulses with 90 ps 


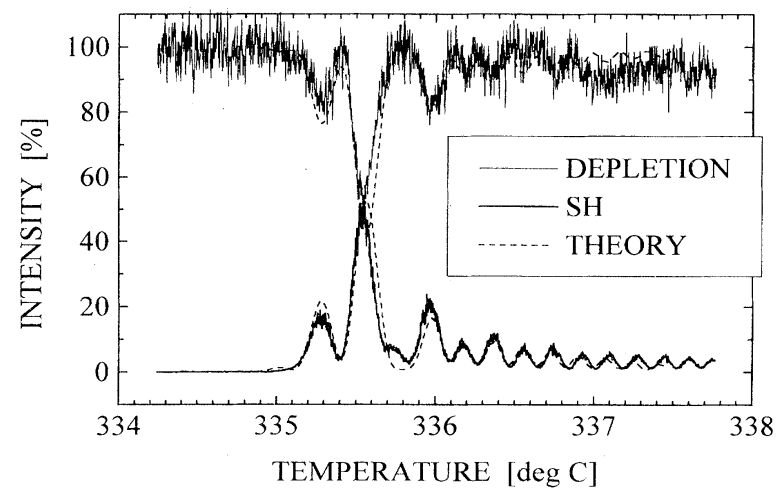

FIG. 3. Temperature dependence of the total fundamental and the second-harmonic outputs for a beam with $94 \mathrm{~W}$ total peak power.

FWHM and a repetition rate of $800 \mathrm{~Hz}$, using a Nd:YAG (where YAG denotes yttrium aluminum garnet) $Q$-switched, mode-locked pulsed laser and an electro-optic single pulse extractor. The intensity profile measurements of the waveguide output were averaged over many shots with a camera and were corrected for the background light (measured separately) due to leakage of the $Q$-switched, mode-locked pulse envelope through the pulse slicer.

The variation in the fundamental throughput and SHG with oven temperature is shown in Fig. 3. Both curves are asymmetric with respect to the phase-matching temperature $\left(T_{\mathrm{PM}}=335.55^{\circ} \mathrm{C}\right)$ due to a nonuniform temperature profile along the waveguide shown in Fig. 1 [15]. The advantage of this nonuniformity is that in the region below $T_{\mathrm{PM}}$, large phase distortions can be obtained with only small fundamental depletion. Modeling based on a Gaussian index profile that is dependent on wavelength, polarization, propagation direction, temperature, and indiffused Ti concentration [16], and taking into account the oven's temperature profile, leads to tuning curves that reproduce the experimental results well, as illustrated in Fig. 3. The modeling is further confirmed by the good agreement between the calculated low-power second-harmonic conversion $P_{2}=16.7 P_{1}^{2}$ and the experimental value of $P_{2}=(16.1 \pm 10 \%) P_{1}^{2}$ at phase matching, where $P_{1}$ is in $\mathrm{mW}$ and $P_{2}$ in $\mu \mathrm{W}$. Losses of $0.17 \mathrm{~dB} / \mathrm{cm}$ for the $\mathrm{TM}_{0}$ mode and $0.35 \mathrm{~dB} / \mathrm{cm}$ for the $\mathrm{TE}_{1}$ mode are included in the calculations.

The generation of spatial solitonlike beams was verified by two separate sets of measurements. By ramping the temperature through phase matching, the wave-vector detuning was varied, which in turn changed the magnitude of the effective nonlinearity, i.e., phase distortion. As specified by the modeling, the input peak power density in the center of the beam was $25 \mathrm{~W} / \mu \mathrm{m}$, corresponding to $1.9 \mathrm{~kW}$ total power in the Gaussian beam with a $70 \mu \mathrm{m}$ width. The beam stabilization into a spatial solitary wave is clear in Fig. 4, where the output beam profiles at four selected temperatures are compared with the input beam profile. Far from phase matching the output beam is diffracted to $\sim 3$ times the width of the input beam. Reduction of the wave-vector-mismatch increases the cascaded nonlinearity, which counteracts diffraction. At $T=334.85{ }^{\circ} \mathrm{C}$, just below $T_{\mathrm{PM}}$ and with less than $2 \%$ harmonic conversion, the out-coupled beam has the same
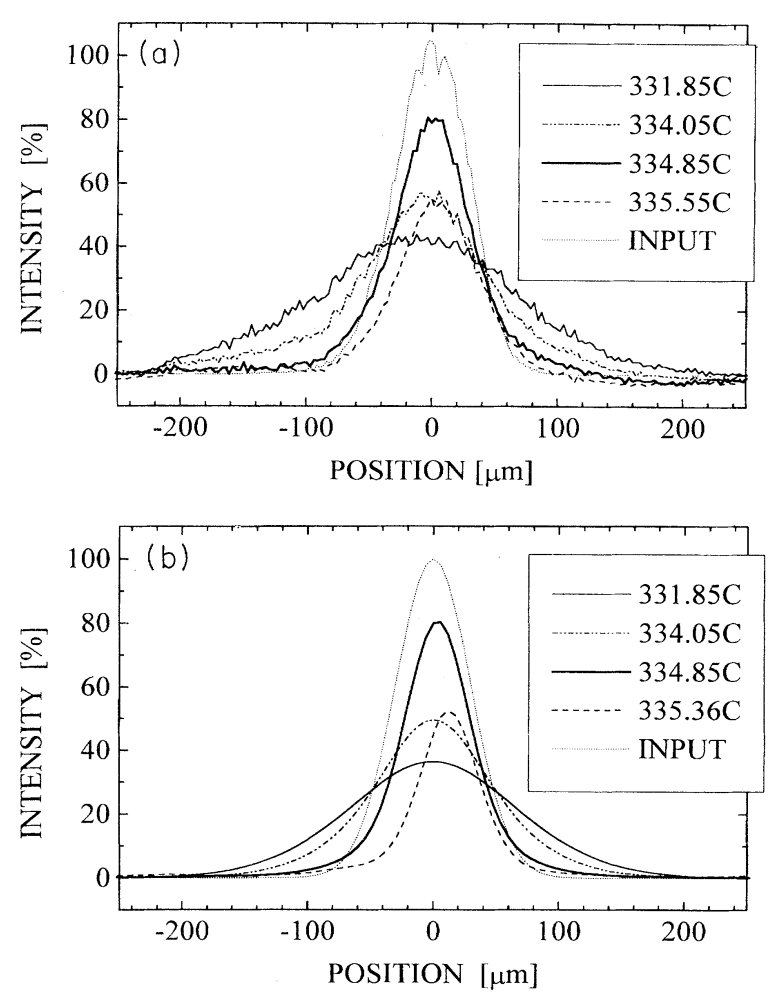

FIG. 4. Temperature dependence of the fundamental beam profile at the out-coupling surface of the waveguide for a beam with $1.9 \mathrm{~kW}$ peak power, (a) experiment and (b) theory.

profile as the input beam, as predicted numerically. The power reduction of $\sim 20 \%$ is due to damping. In this temperature region we take full advantage of the nonuniform wave-vector distribution since here the condition for spatial solitonlike propagation is satisfied in the temperature regime where depletion is minimized and phase shifts are large [15]. In the experiment we needed $20-25 \mathrm{~W} / \mu \mathrm{m}$ peak power density for detecting clean solitary-wave production, in good agreement with the simulations.

At temperatures around $T_{\mathrm{PM}}$ the cascaded nonlinearity is the strongest and a significant amount of power is converted into the second harmonic, which explains the reduced fundamental throughput at $335.55^{\circ} \mathrm{C}$ (Fig. 4). As shown by our modeling and predicted previously with and without secondharmonic seeding, mutual self-trapping of the fundamental and second-harmonic occurs [7-13]. The stronger the SHG, the larger the deviation of the resulting solitary waves from $\chi^{(3)}$-like spatial solitons [Eq. (5)]. Our sample is too short for the interacting fields to stabilize into the asymptotic spatial solitary waves without second-harmonic seeding. However, near $T_{\mathrm{PM}}$ we measured a very interesting feature of cascaded solitary waves. The nonlinear change in the propagation direction indicated by the small position shift of the intensity maximum at $335.55^{\circ} \mathrm{C}$ is a result of the spatial dispersion of the cascaded nonlinearity and was already predicted in [7]. It appears here only because we broke the symmetry by propagating $\sim 1^{\circ}$ off the crystal $X$ axis. The theoretical simulations of the measured intensities are plotted in Fig. 4(b) and the excellent agreement further confirms the model. 


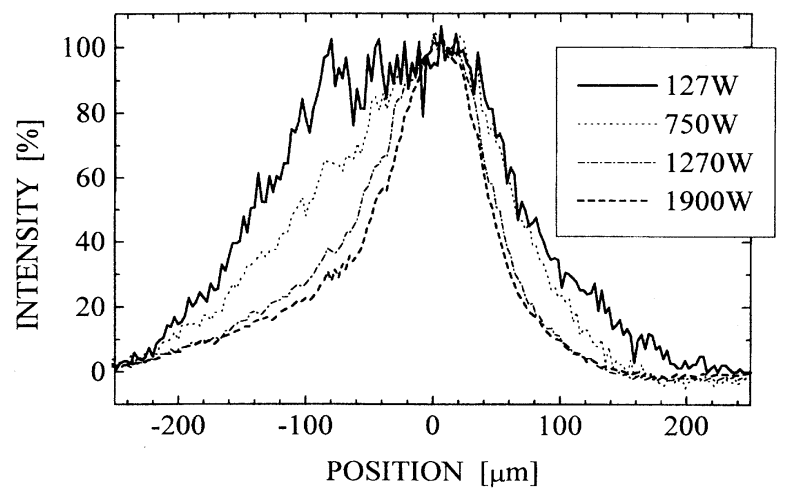

FIG. 5. Normalized output beam profiles at a temperature of $335.00{ }^{\circ} \mathrm{C}$ for beams with a total peak power of $127 \mathrm{~W}, 750 \mathrm{~W}, 1.27$ $\mathrm{kW}$, and $1.9 \mathrm{~kW}$.

We also measured the dependence of the output beam profile on input power at the fixed temperature of $335^{\circ} \mathrm{C}$, where minimum SHG occurs. As can be clearly seen in Fig. 5 , the width of the output beam narrows with increasing power until the width predicted for a solitary wave is reached, and then only changes by at most $10 \%$ out to the largest power studied (intermediate data not shown). These results are all in agreement with our simulations. At the power predicted for optimum solitary-wave generation, the output beam equals the input beam. The data are noisier relative to the temperature scan because of the temperature fluctuations due to the temperature regulator.

Comparable results, again in excellent agreement with theory, were obtained near phase-matched SHG of the fundamental $\mathrm{TM}_{0}$ to the $\mathrm{TE}_{0}$ harmonic at $\sim 290{ }^{\circ} \mathrm{C}$. The two SHG resonances, converted into the $\mathrm{TE}_{0}$ and $\mathrm{TE}_{1}$ modes, are well separated. No nonlinear coupling between them was observed in the data, confirming our approximation that SHG can be considered individually for each process.

In conclusion, we have experimentally observed onedimensional spatial optical solitary waves based on a physical process, the cascaded second-order nonlinearity. We base this conclusion on the excellent, detailed agreement between experiment and theory. As a result, we expect that onedimensional spatial solitary waves should occur under appropriate conditions in other second-order processes, i.e., parametric mixing, optical parametric amplification, etc. By using materials with a higher nonlinear coefficient it will be possible to lower the input power level to that required for a practical solitonlike switching device.

We thank Dr. W. Sohler from the GH-University Paderborn for preparing the excellent waveguide and providing the crystal oven. The research at CREOL was supported by ARPA and the Army Research Office.
[1] See, for example, A. C. Newell, Solitons in Mathematics and Physics (Society for Industrial and Applied Mathematics, Philadelphia, 1985).

[2] L. F. Mollenauer, R. H. Stolen, and J. P. Gorden, Phys. Rev. Lett. 45, 1095 (1980); A. M. Weiner et al., ibid. 61, 2445 (1988).

[3] J. S. Aitchison et al., Opt. Lett. 15, 471 (1990); G. A. Swartzlander et al., Phys. Rev. Lett. 66, 1583 (1991).

[4] See, for example, M. Shalaby, F. Reynaud, and A. Barthelemy, Opt. Lett. 17, 778 (1992).

[5] L. A. Ostrovskii, Pis'ma Zh. Eksp. Teor. Fiz. 5, 331 (1966) [JETP Lett. 5, 272 (1967)].

[6] G. I. Stegeman et al., Opt. Lett. 18, 13 (1993).

[7] R. Schiek, J. Opt. Soc. Am. B 10, 1848 (1993).

[8] Y. N. Karamzin and A. P. Sukhorukov, Pisma Zh. Eksp. Teor. Fiz. 20, 734 (1973) [JETP Lett. 20, 339 (1974)]; Zh. Eksp.
Teor. Fiz. 68, 834 (1975) [Sov. Phys. JETP 41, 414 (1976)].

[9] K. Hayata and M. Koshiba, Phys. Rev. Lett. 71, 3275 (1993).

[10] M. J. Werner and P. D. Drummond, J. Opt. Soc. Am. B 10, 2390 (1993).

[11] L. Torner, C. R. Menyuk, and G. I. Stegeman, Opt. Lett. 19, 1615 (1994).

[12] A. V. Buryak and Y. S. Kivshar, Opt. Lett. 19, 1612 (1994).

[13] R. Schiek, Nonlinear Guided-Wave Phenomena Technical Digest 1993 (Optical Society of America, Washington, DC, 1993), Vol. 15, p. 151.

[14] J. Satsuma and N. Yajima, Suppl. Prog. Theor. Phys. 55, 284 (1974).

[15] R. Schiek et al., Opt. Lett. 19, 1949 (1994).

[16] E. Strake, G. P. Bava, and I. Montrosset, J. Lightwave Technol. 6, 1126 (1988). 